\title{
Comparison of intraoperative frozen section analysis for sentinel lymph node biopsy during breast cancer surgery for invasive lobular carcinoma and invasive ductal carcinoma
}

\author{
James W Horvath ${ }^{1}$, Gary E Barnett ${ }^{1}$, Rafael E Jimenez ${ }^{1}$, Donn C Young ${ }^{2}$ and \\ Stephen P Povoski*3
}

Address: ${ }^{1}$ Department of Pathology, The Ohio State University, Columbus, Ohio 43210, USA, ${ }^{2}$ Center for Biostatistics, The Ohio State University, Columbus, Ohio 43210, USA and ${ }^{3}$ Division of Surgical Oncology, Department of Surgery, Arthur G. James Cancer Hospital and Richard J. Solove Research Institute and Comprehensive Cancer Center, The Ohio State University, Columbus, Ohio 43210, USA

Email: James W Horvath - james.horvath@osumc.edu; Gary E Barnett - gary.barnett@osumc.edu; Rafael E Jimenez - Jimenez.Rafael@mayo.edu; Donn C Young - young.7@osu.edu; Stephen P Povoski* - stephen.povoski@osumc.edu

* Corresponding author

Published: 24 March 2009

World Journal of Surgical Oncology 2009, 7:34 doi:10.1186/1477-7819-7-34

This article is available from: http://www.wjso.com/content/7/I/34

(c) 2009 Horvath et al; licensee BioMed Central Ltd.

This is an Open Access article distributed under the terms of the Creative Commons Attribution License (http://creativecommons.org/licenses/by/2.0), which permits unrestricted use, distribution, and reproduction in any medium, provided the original work is properly cited.
Received: 21 December 2008

Accepted: 24 March 2009

\begin{abstract}
Background: Sentinel lymph node (SLN) biopsy is the standard of care for the surgical assessment of the axilla during breast cancer surgery. However, the diagnostic accuracy of intraoperative frozen section analysis for confirming metastatic involvement of SLNs in cases of invasive lobular carcinoma (ILC) versus that of invasive ductal carcinoma (IDC) has generated controversy secondary to a frequently low-grade cytologic appearance and an often discohesive pattern displayed by metastatic lymph nodes in ILC. In the current report, we present a comparison of intraoperative frozen section analysis for confirming the presence of metastatic disease within SLNs during breast cancer surgery for ILC and IDC.

Methods: We evaluated the results of I3I consecutive cases of ILC from I 997 to 2008 and I33 cases of IDC (selected by a random sequence generator program) from amongst I I 63 consecutive cases of IDC from the same time period. All cases had at least one SLN that had both intraoperative frozen section analysis and confirmatory permanent section analysis performed.

Results: No statistically significant difference was found in the sensitivity ( $67 \%$ vs. $75 \%, P=0.385)$, specificity ( $100 \%$ vs. $100 \%)$, accuracy $(86 \%$ vs. $92 \%, P=0.172)$, false negative rate $(33 \%$ vs. $25 \%, P$ $=0.385)$, negative predictive value $(81 \%$ vs. $89 \%, P=0.158)$, and positive predictive value $(100 \%$ vs. $100 \%$ ) for frozen section analysis for confirming the presence of metastatic disease within SLNs during breast cancer surgery for ILC and IDC.

Conclusion: Since there was no statistically significant difference in sensitivity, specificity, accuracy, false negative rate, negative predictive value, and positive predictive value between frozen section analysis of SLNs for patients with ILC and IDC, the clinical accuracy of confirming metastatic involvement of SLNs on frozen section analysis for ILC should not be considered inferior to the clinical accuracy for IDC. Therefore, frozen section analysis of all SLNs during breast cancer surgery in patients with ILC should remain the standard of care in order to reduce the risk of the need of a later, separate axillary lymph node dissection.
\end{abstract}




\section{Background}

Sentinel lymph node (SLN) biopsy with intraoperative frozen section analysis has become a standard of care in the surgical staging of the axilla during breast cancer surgery [1-3]. The sensitivity of intraoperative frozen section analysis for identifying nodal metastases within SLNs during breast cancer surgery has been reported to vary widely from the range of $44 \%$ to $95 \%$ [4-17], with most series reporting the sensitivity of frozen section analysis in the range of $60 \%$ to $75 \%[5,7-9,11-13,15-17]$.

The difficulty with identifying nodal metastases from invasive lobular carcinoma (ILC) versus invasive ductal carcinoma (IDC) has long been debated within the pathology and surgical communities [17-24]. It has been suggested that individual tumor cells involving the subcapsular sinuses of SLNs in patients with ILC can closely resemble benign lymphocytes and histiocytes when evaluated at frozen section [17,20-24]. Likewise, it has been suggested that the bland cytologic features, round to spindled shape, and discohesive proliferation of ILC cells can make their diagnosis on $\mathrm{H} \& \mathrm{E}$ alone especially difficult $[17,20-22,24]$. Due to this perceived difficulty in identifying metastatic disease within lymph nodes harvested from patients with ILC, it has been suggested by several authors that false negative frozen section results are more likely in SLN biopsy for ILC as compared to IDC $[12,13,17]$.

The rate of nodal positivity of ILC versus IDC has been extensively compared in the literature [25-36], with most studies showing no significant difference $[25,28,30$ $33,35,36]$, and only isolated reports showing a significant difference in nodal positivity favoring more in ILC [26] and favoring more in IDC $[27,29,34]$. Since the diagnostic accuracy of intraoperative frozen section analysis for confirming the presence of metastatic disease within SLNs for ILC versus IDC has long been contended, in the current report, we present a comparison of intraoperative frozen section analysis for confirming the presence of metastatic disease within SLNs during breast cancer surgery for ILC and IDC.

\section{Methods}

\section{Patient selection}

This study was performed under an established Pathology Department protocol approved by Institutional Review Board for the prospectively maintained CoPath database of the Department of Pathology at The Ohio State University.

All female cases of ILC that had undergone frozen section analysis and confirmatory permanent section analysis that was performed on at least one SLN candidate during definitive breast cancer surgery between the time period of 1997 to 2008 were identified from within the CoPath database. This included 131 cases of ILC. From the same time period of 1997 to 2008, all female cases of IDC ( $\mathrm{n}=$ 1163 ) that had undergone intraoperative frozen section analysis and confirmatory permanent section analyses that was performed on at least one SLN during definitive breast cancer surgery were also identified from within the CoPath database. Using an internet-available random sequence generator program called "RANDOM.ORG" [37], a similar number of IDC cases $(n=133)$ were randomly selected from amongst the entire group of IDC cases in order to generate a cohort of IDC cases to be used for direct comparison to the ILC cases.

All female breast cancer cases identified from within the CoPath database that reported mixed lobular/ductal features were excluded from consideration for inclusion in either the ILC group or the IDC group.

\section{Surgical considerations}

The technical details with regards to performing SLN biopsy during breast cancer surgery at The Ohio State University, including the exact methods of injection of radiocolloid and vital blue dye, have been previously described for the time period prior to 2001 [38] and for the time period since 2001 [39].

\section{Histopathology considerations}

At the current time, during intraoperative consultation for frozen section analysis at The Ohio State University, each SLN is grossly sectioned at $0.2 \mathrm{~cm}$ interval portions. The most superficial $25 \%$ of the thickness of each resulting 0.2 $\mathrm{cm}$ SLN tissue section is processed for frozen section analysis, providing at least three separate levels of tissue for frozen section analysis. These frozen sections are then hand-stained by routine Hematoxylin and Eosin (H\&E) staining. The remaining tissue of each resulting $0.2 \mathrm{~cm}$ SLN tissue section, encompassing $75 \%$ of the thickness of that tissue, is then sent for routine processing. Three separate levels (level 1,2, and 3) on permanent slides are then sectioned at approximately $500 \mu \mathrm{m}$ intervals and levels 1 and 3 are stained with $\mathrm{H} \& \mathrm{E}$ by an automated staining device, while level 2 is immunohistochemically stained with cytokeratin AE1/AE3. In those specific cases that are reported as having a SLN that is positive for metastatic carcinoma on the frozen section analysis, the level 2 section from each submitted SLN is omitted from undergoing routine cytokeratin AE1/AE3 immunohistochemistry (IHC). We do recognize that the exact methodology of performing frozen section analysis and permanent histopathologic analysis of SLNs for breast cancer cases has changed during the study period from 1997 to 2008.

Metastatic disease within a sentinel lymph node was defined as "macrometastatic" if any given tumor deposit was greater than $2.0 \mathrm{~mm}$ and was defined as "micrometa- 
static" if any given tumor deposit was less than or equal to $2.0 \mathrm{~mm}$. Due to the fact that the study period extends back to 1997 and due to the fact that there was some degree of variability in the reporting style of the multiple original reading pathologists for each of these cases, it was not feasible to accurately further subclassify micrometastatic disease into "micrometastatic" and "submicrometastatic" subclassifications.

It is important to note that the inception of the performance of routine cytokeratin AE1/AE3 IHC for breast cancer cases in which all of the SLNs were reported as negative at the time of the initial frozen section analysis was initiated at The Ohio State University in May 2006. Before May 2006 , it was specifically at the discretion of the reading pathologist as to whether or not to utilize cytokeratin AE1/AE3 IHC for further and for more in-depth evaluation of SLNs in any given breast cancer case. Therefore, since it would be difficult to assess the impact of cytokeratin AE1/AE3 IHC on the overall results reported in the current study secondary to the obvious heterogeneity of the application of cytokeratin AE1/AE3 IHC from 1997 through 2006, no attempt was made to differentiate the results of permanent pathologic evaluation based upon whether cytokeratin AE1/AE3 IHC was used or not used.

\section{Data collection and analyses}

Multiple patient variables, primary tumor variables, and SLN variables were evaluated for each case. Data collection of all those variables was simply accomplished by way of retrospective review of the electronic pathology report posted by the original reading pathologist for each case. A re-review of the actual H\&E frozen section slides, $H \& E$ permanent section slides, and cytokeratin IHC slides for these cases was not undertaken as part of the current analysis. If a given variable was absent from the electronic pathology report posted by the original reading pathologist, that variable was recorded as unknown for that particular case.

The number of true positive (TP), true negative (TN), false negative (FN), and false positive (FP) were determined for frozen section analysis compared to permanent section analysis for the finding of positive SLN for ILC versus IDC. Then, for both ILC and IDC, the sensitivity $(\mathrm{TP} /(\mathrm{TP}+\mathrm{FN}))$, specificity (TN/(TN+FP)), accuracy $((\mathrm{TP}+\mathrm{TN}) /$ total patients), false negative rate $(\mathrm{FN} /(\mathrm{TP}+\mathrm{FN}))$, negative predictive value $(\mathrm{TN} /(\mathrm{TN}+\mathrm{FN}))$, and positive predictive value $(\mathrm{TP} /(\mathrm{TP}+\mathrm{FP}))$ were calculated. All these variables were determined on a per patient basis and were not determined on a per SLN basis.

The software program SPSS 16.0 for Windows (SPSS, Inc., Chicago, Illinois) was used for all statistical analyses. For univariate comparisons of categorical variables, either
Pearson chi-square test or Fisher exact test was utilized. Continuous variables were expressed as median (range). For univariate comparisons of continuous variables, oneway analysis of variance (ANOVA) was utilized. All reported univariate P-values were two-sided. All univariate P-values determined to be 0.05 or less were considered to be statistically significant.

\section{Results}

Patient and tumor demographics for ILC and IDC patients are shown in Table 1. ILC patients tended to be older. ILC patients generally had larger tumors and more often displayed multifocal and multicentric disease. ILC generally had a lower histologic tumor grade and were more often estrogen receptor positive, progesterone receptor positive, and Her-2/neu negative. ILC less often had displayed lymphovascular invasion.

The SLN demographics, including frozen section analysis results, permanent section analysis results, the size of the SLN metastasis, and the classification into macrometastatic disease and micrometastatic disease for ILC and IDC patients are shown in Table 2. No statistically significant difference was noted in any of these SLN demographics variables for ILC versus IDC patients.

The number of TP, TN, FN, and FP were determined for frozen section analysis compared to permanent section analysis for the finding of a positive SLN for ILC versus IDC patients and are shown in Table 3 . The nature of the classification of metastatic disease (i.e., macrometastatic versus micrometastatic) amongst false negative cases for patients with a positive sentinel lymph node for ILC versus IDC is shown in Table 4. No statistically significant difference was noted in any of these variables for ILC versus IDC patients.

The sensitivity, specificity, accuracy, false negative rate, negative predictive value, and positive predictive value of frozen section analysis compared to permanent section analysis for the finding of positive SLN for ILC versus IDC patients were calculated and are shown in Table 5. No statistically significant difference was noted in any of these variables for ILC versus IDC patients.

\section{Discussion}

The primary reason for undertaking this current analysis was the fact that it has been the longstanding general opinion of many surgical pathologists within the pathology community, including our own, that SLNs in ILC cases are notoriously more difficult to interpret, especially at the time of frozen section analysis. This longstanding contention has been eloquently addressed and debated within the literature [18-24]. Best articulated by Creager et al [21], although not necessarily agreed upon by their 
Table I: Patient and tumor demographics for invasive lobular carcinomas and invasive ductal carcinomas

\begin{tabular}{|c|c|c|c|c|}
\hline & $\operatorname{ILC}(n=|3|)$ & IDC $(n=133)$ & Total cases $(n=264)$ & P-value \\
\hline Age (years) & $59(35-87)$ & $55(24-82)$ & $57(24-87)$ & 0.001 \\
\hline Tumor size $(\mathrm{cm})$ & $2.0(0.2-9.0)$ & $1.5(0.1-6.5)$ & I.7 (0.1-9.0) & 0.006 \\
\hline \multicolumn{5}{|l|}{ T-stage } \\
\hline $\mathrm{TI}$ & $75(58 \%)$ & $88(66 \%)$ & 163 (62\%) & 0.298 \\
\hline $\mathrm{T} 2$ & $49(38 \%)$ & 43 (32\%) & $92(35 \%)$ & \\
\hline T3 & $5(4 \%)$ & $2(2 \%)$ & $7(3 \%)$ & \\
\hline $\mathrm{T} 4$ & I (1\%) & $0(0 \%)$ & $\mathrm{I}(0.5 \%)$ & \\
\hline \multicolumn{5}{|l|}{ Tumor focality } \\
\hline Unifocal & 97 (75\%) & $123(93 \%)$ & $220(84 \%)$ & $<0.001$ \\
\hline Multifocal & $19(15 \%)$ & $9(7 \%)$ & $28(11 \%)$ & \\
\hline Multicentric & 14 (II\%) & I (I\%) & $15(6 \%)$ & \\
\hline \multicolumn{5}{|l|}{ Histologic grade } \\
\hline Grade I & 40 (31\%) & $32(24 \%)$ & 72 (27\%) & $<0.001$ \\
\hline Grade 2 & 57 (44\%) & 52 (39\%) & $109(4 \mid \%)$ & \\
\hline Grade 3 & $17(13 \%)$ & $48(36 \%)$ & $65(25 \%)$ & \\
\hline Unknown & 17 (13\%) & I (I\%) & $18(7 \%)$ & \\
\hline \multicolumn{5}{|l|}{ ER status } \\
\hline Positive & $12 \mid(93 \%)$ & $96(72 \%)$ & $217(82)$ & $<0.001$ \\
\hline Negative & $2(2 \%)$ & $29(22 \%)$ & $31(12 \%)$ & \\
\hline Unknown & $8(6 \%)$ & $8(6 \%)$ & $16(6 \%)$ & \\
\hline \multicolumn{5}{|l|}{ PR status } \\
\hline Positive & $108(82 \%)$ & 81 (61\%) & $189(72 \%)$ & $<0.001$ \\
\hline Negative & $16(12 \%)$ & $44(33 \%)$ & $60(23 \%)$ & \\
\hline Unknown & $7(5 \%)$ & $8(6 \%)$ & $15(6 \%)$ & \\
\hline \multicolumn{5}{|l|}{ Her-2/neu } \\
\hline Positive & $13(10 \%)$ & $35(26 \%)$ & $48(18 \%)$ & 0.003 \\
\hline Negative & $106(81 \%)$ & 87 (65\%) & $193(73 \%)$ & \\
\hline Unknown & $12(9 \%)$ & II (8\%) & $23(9 \%)$ & \\
\hline \multicolumn{5}{|l|}{ LVI } \\
\hline Positive & $20(15 \%)$ & $4 \mid(3 \mid \%)$ & 61 (23\%) & 0.005 \\
\hline Negative & $109(83 \%)$ & 92 (69\%) & $201(76 \%)$ & \\
\hline Unknown & $2(2 \%)$ & $0(0 \%)$ & $2(1 \%)$ & \\
\hline
\end{tabular}

ILC, invasive lobular carcinoma; IDC, invasive ductal carcinoma; ER, estrogen receptor; PR, progesterone receptor; LVI, lymphovascular invasion

group, this longstanding contention specifically asserts that the intraoperative detection of ILC can be highly problematic secondary to its low-grade cytomorphology and its tendency to infiltrate metastatic sites in a single cell pattern. This assertion that was articulated by Creager et al [21] suggests that such architectural and cytomorphologic features of ILC within a given metastatic lymph node can result in occasionally missing even relatively large nodal metastases on intraoperative frozen section evaluation that are then only discovered, much to the surprise of the pathologist and surgeon, on permanent $H \& E$ sections and/or cytokeratin AE1/AE3 IHC stained sections. In this regard, our goal was to compare the diagnostic accuracy of intraoperative frozen section analysis for confirming the presence of metastatic disease within SLNs during breast cancer surgery for ILC and IDC, in order to confirm or dispel the above, longstanding contention.

In our study, the sensitivity of frozen section analysis (67\% for ILC patients, $75 \%$ for IDC patients, and $70 \%$ for all patients) was well within the range of sensitivity for frozen section analysis results (i.e. $60 \%$ to $75 \%$ range) in most previously reported series in the literature for SLN biopsy during breast cancer surgery [5,7-9,11-13,15-17]. Therefore, our frozen section analysis results, based on sensitivity, are highly consistent with the mainstream practice of intraoperative frozen section analysis for SLN biopsy during breast cancer surgery. 
Table 2: The sentinel lymph node demographics for invasive lobular carcinomas and invasive ductal carcinomas

\begin{tabular}{|c|c|c|c|c|}
\hline & ILC & IDC & Total cases & P-value \\
\hline \multicolumn{5}{|l|}{ Frozen section analysis of SLN } \\
\hline Positive & $36(28 \%)$ & $33(25 \%)$ & $69(26 \%)$ & 0.622 \\
\hline Negative & $95(73 \%)$ & $100(75 \%)$ & $195(74 \%)$ & \\
\hline \multicolumn{5}{|l|}{ Permanent section analysis of SLN } \\
\hline Positive & $54(4 \mid \%)$ & 44 (33\%) & 98 (37\%) & 0.171 \\
\hline Negative & 77 (59\%) & $89(67 \%)$ & $166(63 \%)$ & \\
\hline Size of SLN metastasis $(\mathrm{mm})$ & $6.0(0.5-25.0)$ & $5.0(0.1-32.0)$ & $5.5(0.1-32.0)$ & 0.808 \\
\hline \multicolumn{5}{|l|}{ Classification of metastatic disease } \\
\hline Macrometastatic $(>2.0 \mathrm{~mm})$ & $36(28 \%)$ & $28(21 \%)$ & $64(24 \%)$ & 0.378 \\
\hline Micrometastatic $(\leq 2.0 \mathrm{~mm})$ & $16(12 \%)$ & $12(9 \%)$ & $28(11 \%)$ & \\
\hline Unknown classification & $2(1.5 \%)$ & $4(3 \%)$ & $6(2 \%)$ & \\
\hline No metastatic disease & 77 (59\%) & $89(67 \%)$ & $166(63 \%)$ & \\
\hline
\end{tabular}

ILC, invasive lobular carcinoma; IDC, invasive ductal carcinoma; SLN, sentinel lymph node

Likewise, in our study, we did not find a statistically significant difference in the false negative rate for frozen section analysis for SLN biopsy for ILC as compared to IDC (33\% for ILC, $25 \%$ for IDC, $\mathrm{P}=0.385$ ). Although this may initially seem surprising to some, the vast majority of the literature supports the routine use of intraoperative frozen section analysis for SLN biopsy during breast cancer surgery for ILC cases $[10,12,13,16,21,24,40,41]$. Nevertheless, several authors have previously reported that false negative frozen section results are more likely in SLN biopsy for ILC as compared to for IDC $[12,13,17]$.

Leidenius et al [12] analyzed a total of 375 breast cancers and reported that the false-negative rate for frozen section analysis during SLN biopsy was more common for ILC than IDC $(28 \%$ versus $8 \%, \mathrm{P}<0.01)$ in an overall analysis of 102 ILC versus 194 IDC. In our estimation, the distribution of tumor types (i.e., ILC versus IDC) reported by Leidenius et al [12] is very perplexing. In their series [12], they reported seeing 102 cases of ILC among a total of 375

\begin{tabular}{|c|c|c|c|c|}
\hline & ILC & IDC & Total cases & P-value \\
\hline TP & 36 & 33 & 69 & 0.622 \\
\hline $\mathrm{TN}$ & 77 & 89 & 166 & 0.171 \\
\hline $\mathrm{FN}$ & 18 & 11 & 29 & 0.155 \\
\hline FP & 0 & 0 & 0 & ------ \\
\hline Total & $|3|$ & 133 & 264 & \\
\hline
\end{tabular}

ILC, invasive lobular carcinoma; IDC, invasive ductal carcinoma; TP, true positive; TN, true negative; FP, false positive; FN, false negative total breast cancer cases during a 22 month time period from $01 / 02 / 2001$ to $11 / 7 / 2002$ in Helsinki, Finland. This signifies that ILC makes up an astonishing $27.2 \%$ of all the breast cancers seen in Helsinki, Finland. This is in stark contrast to the maximum of $10 \%$ to $15 \%$ of ILC cases that are generally seen among all presenting breast cancers within the United States $[17,26,34,35]$ and worldwide $[27,30,31,33,36,41,42]$. Secondly, they found an unusually low false negative rate of frozen section analysis for SLNs for IDC cases (8\%) as compared to for ILC cases (28\%) [12]. In contrast, most series in the literature generally report a false negative rate of frozen section analysis for SLN biopsy for breast cancer cases is in the range of anywhere from $26 \%$ to $56 \%[5,7,8,10,11,13-15,17]$, including our own current series in which the false negative rate of frozen section analysis for SLN biopsy was $33 \%$ for ILC, $25 \%$ for IDC, and 30\% for all breast cancer cases. This particular aspect of Leidenius et al [12] reported series can not be easily explained in view of the rest of the reported literature and casts some doubt into their results and contention that false negative frozen section results are more likely in SLN biopsy for ILC as compared to for IDC.

Similarly, Holck et al [13] analyzed a total of 265 breast cancers and reported that false negative findings were overrepresented for ILC on frozen section analysis during SLN biopsy (i.e., 5 of 28 or $17.9 \%$ of the false negative frozen section results were from ILC). Despite the fact that Holck et al [13] made this statement, they failed to specify within their paper exactly how many ILC cases they analyzed from among the 265 breast cancers they saw in Hilleroed, Denmark over a 20 month period of time from February 2001 through September 2002 and did not provide enough raw data or P-values to verify their claim for overrepresentation. 
Table 4: The nature of the classification of metastatic disease amongst false negative cases for patients with a positive sentinel lymph node for invasive lobular carcinomas and invasive ductal carcinomas

\begin{tabular}{llll}
\hline False negative cases & ILC & IDC & Total cases \\
\hline Macrometastatic $(>2.0 \mathrm{~mm})$ & $5(28 \%)$ & $2(18 \%)$ & $7(24 \%)$ \\
Micrometastatic $(\leq 2.0 \mathrm{~mm})$ & $11(61 \%)$ & $8(73 \%)$ & $19(66 \%)$ \\
Unknown classification & $2(11 \%)$ & $1(9 \%)$ & $3(10 \%)$ \\
\hline Total & $18(100 \%)$ & $11(100 \%)$ & $29(100 \%)$ \\
\hline
\end{tabular}

ILC, invasive lobular carcinoma; IDC, invasive ductal carcinoma

Lastly, Chan et al [17] most recently analyzed a total of 5298 breast cancers and reported that the false negative rate for frozen section analysis during SLN biopsy was more common for ILC than IDC (47.6\% versus $37.8 \%$, P $=0.006)$ in an overall analysis of 574 ILC versus 4531 IDC. Despite the statistically significant difference between ILC and IDC that they reported, Chan et al [17] went on to state in their discussion that "although this difference is statistically significant, it may not be clinically significant, as frozen section successfully detected a majority of SLN metastases in both groups". Likewise, Chan et al [17] never concluded in their report that frozen section analysis of SLNs during breast cancer surgery for ILC should be abandoned.

Therefore, it is reasonable to conclude, based on our results showing no significant statistical difference in the false negative rate on frozen section analysis for SLNs in ILC versus IDC cases, that intraoperative frozen section analysis of SLNs during breast cancer surgery for ILC should remain an important standard of care. This allows for accurate intraoperative assessment of the nodal status of the axilla, thus allowing the surgeon to appropriately proceed with an immediate concomitant axillary lymph node dissection based upon the intraoperative finding of a positive SLN and thus minimizing the need for an additional, subsequent, delayed axillary procedure. Clearly, intraoperative frozen section analysis during SLN biopsy is no less important for ILC than it is for IDC.

Table 5: The sensitivity, specificity, accuracy, false negative rate, negative predictive value, and positive predictive value of frozen section analysis compared to permanent section analysis for the finding of a positive sentinel lymph node for invasive lobular carcinomas and invasive ductal carcinomas

\begin{tabular}{lllll}
\hline & ILC & IDC & Total cases & P-value \\
\hline Sensitivity & $67 \%$ & $75 \%$ & $70 \%$ & 0.385 \\
Specificity & $100 \%$ & $100 \%$ & $100 \%$ & ----- \\
Accuracy & $86 \%$ & $92 \%$ & $89 \%$ & 0.172 \\
False negative rate & $33 \%$ & $25 \%$ & $30 \%$ & 0.385 \\
Negative predictive value & $81 \%$ & $89 \%$ & $85 \%$ & 0.158 \\
Positive predictive value & $100 \%$ & $100 \%$ & $100 \%$ & -----
\end{tabular}

ILC, invasive lobular carcinoma; IDC, invasive ductal carcinoma
Despite the fact that our results do not show any significant difference in the diagnostic accuracy of intraoperative frozen section analysis using hand-stained, routine $\mathrm{H} \& \mathrm{E}$ staining for confirming the presence of metastatic disease within SLN candidates during breast cancer surgery for ILC and IDC, several relevant issues with regards to the potential impact of IHC on the detection rate of axillary lymph node metastases in cases of ILC seem to be worth further discussion.

Recently, Tan et al [43] retrospectively analyzed a cohort of 368 previously presumed node-negative breast cancer patients (319 with IDC and 49 with ILC) that were treated with axillary lymph node dissection between 1976 and 1978 and who had 20-year follow up. They retrospectively performed IHC in order to attempt to identify occult axillary lymph node metastases based upon IHC detection versus historical standard H\&E detection. From their retrospective performance of IHC, they were able to identify three very important pathological features that were specifically attributable to ILC cases. First, ILC cases had a higher rate of conversion from node negative to node positive than did IDC cases (40\% versus $20 \%$ ). Second, ILC cases had an over-representation IHC-detected disease versus $H \& E$-detected disease (36\% versus $15 \%$ ). Third, ILC cases had an over-representation among patients with single-cell metastases versus clustered metastases $(59 \%$ versus 7\%). Certainly, these pathological features demonstrate the potential impact that IHC may have on the overall diagnostic accuracy of confirming the presence of metastatic disease within SLN candidates for ILC cases. Nevertheless, since this study cohort [43] represents a group of patients treated in the pre-SLN biopsy era, these IHC results have no direct correlation to or bearing upon the current intraoperative assessment of frozen section analysis for confirming the presence of metastatic disease within SLN candidates during breast cancer surgery for ILC.

More relevant to the SLN biopsy era, Patil and Susnik [24] recently retrospectively reviewed 76 patients with ILC undergoing SLN biopsy during the time period of 2003 to 2007. Of the 76 cases, 24 cases (32\%) were positive for metastatic disease (21 macrometastatic and three 
micrometastatic), and 14 cases (18\%) demonstrated isolated tumor cells (submicrometastatic) on IHC only. All macrometastatic cases $(\mathrm{n}=21)$ and two of three micrometastatic cases were identified on standard $\mathrm{H} \& \mathrm{E}$ evaluation alone. All cases of isolated tumor cells $(n=14)$ and one micrometastatic case were detected on IHC alone. Therefore, based on IHC, they officially changed the axillary lymph node status from negative to positive in only one case of micrometastatic disease. They concluded that upstaging very rarely occurred with the use of IHC [24]. Likewise, they concluded that yielding a diagnosis of isolated tumor cells, which prognostically is not completely understood at this time, rarely results in any deviation of the treatment plan and provides no additional advantage over that of a thorough standard H\&E evaluation [24].

A last relevant point of discussion with regards to IHC is that several groups have advocated the specific use of rapid intraoperative IHC in addition to frozen section H\&E stained levels and possibly touch imprints cytology. Leikola et al [23] analyzed 995 breast cancer patients (523 with IDC and 245 with ILC) undergoing SLN biopsy during the time period of 2001 to 2007. They demonstrated that rapid intraoperative IHC on frozen sections analysis improved the sensitivity of detecting metastatic disease within SLNs from $66 \%$ (without IHC) to $87 \%$ (with IHC) for patients with ILC $(\mathrm{P}=0.02)$. Similarly, Weinberg et al [22] analyzed 59 breast cancer patients with ILC using rapid intraoperative IHC on touch imprint cytology. They demonstrated that their sensitivity for identifying a SLN containing metastatic disease was increased from $41.9 \%$ (without IHC) to $54.8 \%$ (with IHC) using rapid intraoperative IHC on touch imprint cytology and concluded that rapid intraoperative IHC on touch imprint cytology enhances the intraoperative diagnosis of SLN metastases in patients with ILC. However, no specific P-values were reported by Weinberg et al [22] to support their data or their conclusions.

While IHC is currently widely utilized at many institutions around the globe as part of standard histopathologic evaluation of SLNs for breast cancer, the specific relevance and impact of IHC can not be directly addressed within the context of the findings of our current report, since we did not specifically analyze IHC findings as an independent variable within our overall assessment of the diagnostic accuracy of intraoperative frozen section analysis for confirming the presence of metastatic disease within SLNs candidates during breast cancer surgery for ILC and IDC. Obviously, the specific impact of IHC on the overall assessment of the diagnostic accuracy of intraoperative frozen section analysis for confirming the presence of metastatic disease is multifactorial and is beyond the scope of our current discussion.

\section{Conclusion}

Since there was no statistically significant difference in sensitivity, specificity, accuracy, false negative rate, negative predictive value, and positive predictive value between frozen section analysis of SLNs for patients with ILC and IDC, the clinical accuracy of confirming metastatic involvement of SLNs on frozen section analysis for ILC should not be considered inferior to the clinical accuracy for IDC. Therefore, frozen section analysis of all SLNs during breast cancer surgery in patients with ILC should remain the standard of care in order to reduce the risk of the need of a later, separate axillary lymph node dissection.

\section{Abbreviations}

SLN: sentinel lymph node; ILC: invasive lobular carcinoma; IDC: invasive ductal carcinoma; IHC: immunohistochemistry; ER: estrogen receptor; PR: progesterone receptor; LVI: lymphovascular invasion; TP: true positive; $\mathrm{TN}$ : true negative; FN: false negative; FP: false positive

\section{Competing interests}

The authors declare that they have no competing interests.

\section{Authors' contributions}

JWH was involved in the study design, data collection, and writing and editing all aspects of this manuscript. GEB and REJ were involved in the study design and editing this manuscript. DCY was involved in the study design, data analysis, and editing this manuscript. SPP was involved in the study design, data analysis, writing and editing all aspects of this manuscript, and represented the senior physician overseeing the project. All of the authors have read and approved the final version of this manuscript.

\section{References}

I. Burak WE, Agnese DM, Povoski SP: Advances in the surgical management of early stage invasive breast cancer. Curr Probl Surg 2004, 41:877-936.

2. National Comprehensive Cancer Network (NCCN) Clinical Guidelines in Oncology for Breast Cancer (V.I.2009) [http:I /www.nccn.org/professionals/physician gls/PDF/breast.pdf]

3. Goyal A, Mansel RE: Recent advances in sentinel lymph node biopsy for breast cancer. Curr Opin Oncol 2008, 20:621-626.

4. Flett MM, Going JJ, Stanton PD, Cooke TG: Sentinel node localization in patients with breast cancer. $B r J$ Surg 1998, 85:991-993.

5. Dixon JM, Mamman U, Thomas J: Accuracy of intraoperative frozen-section analysis of axillary nodes. Edinburgh Breast Unit team. BrJ Surg 1999, 86:392-395.

6. Van Diest PJ, Torrenga H, Borgstein PJ, Pijpers R, Bleichrodt RP, Rahusen FD, Meijer S: Reliability of intraoperative frozen section and imprint cytological investigation of sentinel lymph nodes in breast cancer. Histopathology 1999, 35:14-18.

7. Weiser MR, Montgomery LL, Susnik B, Tan LK, Borgen PI, Cody HS: Is routine intraoperative frozen-section examination of sentinel lymph nodes in breast cancer worthwhile? Ann Surg Oncol 2000, 7:65I-655.

8. Chao C, Wong SL, Ackermann D, Simpson D, Carter MB, Brown CM, Edwards MJ, McMasters KM: Utility of intraoperative frozen sec- 
tion analysis of sentinel lymph nodes in breast cancer. Am J Surg 200I, I 82:609-6I5.

9. Zurrida S, Mazzarol G, Galimberti V, Renne G, Bassi F, lafrate F, Viale $G$ : The problem of the accuracy of intraoperative examination of axillary sentinel nodes in breast cancer. Ann Surg Oncol 200I, 8:8I7-820

10. Gulec SA, Su J, O'Leary JP, Stolier A: Clinical utility of frozen section in sentinel node biopsy in breast cancer. Am Surg 200I, 67:529-532.

I I. Povoski SP, Dauway EL, Ducatman BS: Sentinel lymph node mapping and biopsy for breast cancer at a rural-based university medical center: initial experience with intraparenchymal and intradermal injection routes. Breast Cancer 2002, 9: I 34- I 44.

12. Leidenius MH, Krogerus LA, Toivonen TS, Von Smitten KJ: The feasibility of intraoperative diagnosis of sentinel lymph node metastases in breast cancer. J Surg Oncol 2003, 84:68-73.

13. Holck S, Galatius H, Engel U, Wagner F, Hoffmann J: False-negative frozen section of sentinel lymph node biopsy for breast cancer. Breast 2004, 13:42-48.

14. Wada N, Imoto S, Hasebe T, Ochiai A, Ebihara S, Moriyama N: Evaluation of intraoperative frozen section diagnosis of sentinel lymph nodes in breast cancer. Jpn J Clin Oncol 2004, 34: I I3- I I 7.

15. Mitchell ML: Frozen section diagnosis for axillary sentinel lymph nodes: the first six years. Mod Pathol 2005, I 8:58-6I.

16. Arora N, Martins D, Huston TL, Christos P, Hoda S, Osborne MP, Swistel AJ, Tousimis E, Pressman PI, Simmons RM: Sentinel node positivity rates with and without frozen section for breast cancer. Ann Surg Oncol 2008, I 5:256-26I.

17. Chan SW, LaVigne KA, Port ER, Fey JV, Brogi E, Borgen PI, Cody HS 3rd: Does the benefit of sentinel node frozen section vary between patients with invasive duct, invasive lobular, and favorable histologic subtypes of breast cancer? Ann Surg 2008, 247:|43-149.

18. Bussolati G, Gugliotta P, Morra I, Pietribiasi F, Berardengo E: The immunohistochemical detection of lymph node metastases from infiltrating lobular carcinoma of the breast. $\mathrm{Br} J$ Cancer 1986, 54:63|-636.

19. Trojani M, de Mascarel I, Coindre JM, Bonichon F: Micrometastases to axillary lymph nodes from invasive lobular carcinoma of breast: detection by immunohistochemistry and prognostic significance. Br J Cancer 1987, 56:838-839.

20. Grube BJ, Hansen NM, Ye X, Giuliano AE: Tumor characteristics predictive of sentinel node metastases in 105 consecutive patients with invasive lobular carcinoma. Am J Surg 2002 I 84:372-376.

21. Creager AJ, Geisinger KR, Perrier ND, Shen P, Shaw JA, Young PR, Case D, Levine EA: Intraoperative imprint cytologic evaluation of sentinel lymph nodes for lobular carcinoma of the breast. Ann Surg 2004, 239:61-66.

22. Weinberg ES, Dickson D, White L, Ahmad N, Patel J, Hakam A, Nicosia S, Dupont E, Furman B, Centeno B, Cox C: Cytokeratin staining for intraoperative evaluation of sentinel lymph nodes in patients with invasive lobular carcinoma. Am J Surg 2004, I 88:419-422.

23. Leikola JP, Toivonen TS, Krogerus LA, von Smitten KA, Leidenius MH: Rapid immunohistochemistry enhances the intraoperative diagnosis of sentinel lymph node metastases in invasive lobular breast carcinoma. Cancer 2005, 104:14-19.

24. Patil DT, Susnik B: Keratin immunohistochemistry does not contribute to correct lymph node staging in patients with invasive lobular carcinoma. Hum Pathol 2008, 39:1011-1017.

25. Silverstein MJ, Lewinsky BS, Waisman JR, Gierson ED, Colburn WJ, Senofsky GM, Gamagami P: Infiltrating lobular carcinoma. Is it different from infiltrating duct carcinoma? Cancer 1994, 73:1673-1677.

26. Yeatman TJ, Cantor AB, Smith TJ, Smith SK, Reintgen DS, Miller MS, Ku NN, Baekey PA, Cox CE: Tumor biology of infiltrating lobular carcinoma. Implications for management. Ann Surg 1995, 222:549-559.

27. Sastre-Garau X, Jouve M, Asselain B, Vincent-Salomon A, Beuzeboc P, Dorval T, Durand JC, Fourquet A, Pouillart P: Infiltrating lobular carcinoma of the breast. Clinicopathologic analysis of 975 cases with reference to data on conservative therapy and metastatic patterns. Cancer 1996, 77:1 13-120.

28. Casolo P, Raspadori A, Drei B, Amuso D, Mosca D, Amorotti C, Di Blasio P, De Maria R, De Luca G, Colli G, Ganz E: [Natural history of breast cancer: lobular carcinoma versus ductal carcinoma in our experience]. Ann Ital Chir 1997, 68:43-47. discussion 48. [Italian]

29. Toikkanen S, Pylkkänen L, Joensuu H: Invasive lobular carcinoma of the breast has better short- and long-term survival than invasive ductal carcinoma. Br J Cancer 1997, 76: I234-I240.

30. Mersin H, Yildirim E, Gülben K, Berberoğlu U: Is invasive lobular carcinoma different from invasive ductal carcinoma? Eur J Surg Oncol 2003, 29:390-395.

31. Paumier A, Sagan C, Campion L, Fiche M, Andrieux N, Dravet $F$ Pioud R, Classe JM: [Accuracy of conservative treatment for infiltrating lobular breast cancer: a retrospective study of 217 infiltrating lobular carcinomas and 2 I 55 infiltrating ductal carcinomas]. J Gynecol Obstet Biol Reprod (Paris) 2003, 32:529-534.

32. Korhonen T, Huhtala H, Holli K: A comparison of the biological and clinical features of invasive lobular and ductal carcinomas of the breast. Breast Cancer Res Treat 2004, 85:23-39.

33. Molland JG, Donnellan M, Janu NC, Carmalt HL, Kennedy CW, Gillett DJ: Infiltrating lobular carcinoma-a comparison of diagnosis, management and outcome with infiltrating duct carcinoma. Breast 2004, I 3:389-396.

34. Santiago RJ, Harris EE, Oin L, Hwang WT, Solin LJ: Similar longterm results of breast-conservation treatment for Stage I and II invasive lobular carcinoma compared with invasive ductal carcinoma of the breast: The University of Pennsylvania experience. Cancer 2005, 1 03:2447-2454.

35. Vo TN, Meric-Bernstam F, Yi M, Buchholz TA, Ames FC, Kuerer HM, Bedrosian I, Hunt KK: Outcomes of breast-conservation therapy for invasive lobular carcinoma are equivalent to those for invasive ductal carcinoma. Am / Surg 2006, 192:552-555.

36. Jayasinghe UW, Bilous AM, Boyages J: Is survival from infiltrating lobular carcinoma of the breast different from that of infiltrating ductal carcinoma? Breast / 2007, I 3:479-485.

37. Website operated by Mads Haahr of the School of Computer Science and Statistics at Trinity College, Dublin in Ireland (๑/998-2008 Mads Haahr) [http://www.random.org/ sequences/]

38. Zervos EE, Burak WE Jr: Lymphatic mapping for breast cancer: experience at The Ohio State University. Breast Cancer 2000, 7:195-200.

39. Povoski SP, Olsen JO, Young DC, Clarke J, Burak WE, Walker MJ, Carson WE, Yee LD, Agnese DM, Pozderac RV, Hall NC, Farrar WB: Prospective randomized clinical trial comparing intradermal, intraparenchymal, and subareolar injection routes for sentinel lymph node mapping and biopsy in breast cancer. Ann Surg Oncol 2006, I3:|4|2-|421.

40. Schwartz GF, Krill LS, Palazzo JP, Dasgupta A: Value of intraoperative examination of axillary sentinel nodes in carcinoma of the breast. J Am Coll Surg 2008, 207:758-762.

4I. Classe JM, Loussouarn D, Campion L, Fiche M, Curtet C, Dravet F, Pioud R, Rousseau C, Resche I, Sagan C: Validation of axillary sentinel lymph node detection in the staging of early lobular invasive breast carcinoma: a prospective study. Cancer 2004, 100:935-94I.

42. Martinez V, Azzopardi JG: Invasive lobular carcinoma of the breast: incidence and variants. Histopathology 1979, 3:467-488.

43. Tan LK, Giri D, Hummer AJ, Panageas KS, Brogi E, Norton L, Hudis C. Borgen PI, Cody HS 3rd: Occult axillary node metastases in breast cancer are prognostically significant: results in 368 node-negative patients with 20-year follow-up. J Clin Oncol 2008, 26: 1803-1809. 